\title{
Is uni-portal video-assisted thoracic surgery a feasible approach for the surgical treatment of bronchopulmonary sequestration?
}

\author{
Zhixin $\mathrm{Li}^{1 \#}$, Yongqiang Zhao ${ }^{2 \#}$, Xuefei $\mathrm{Hu}^{1}$, Wenxin $\mathrm{He}^{1}$, Xiaogang Zhao ${ }^{1}$ \\ ${ }^{1}$ Department of Thoracic Surgery, Shanghai Pulmonary Hospital, Tongji University, Shanghai 200433, China; ${ }^{2}$ Department of Thoracic Surgery, \\ Linqu County People's Hospital, Weifang 262600, China \\ Contributions: (I) Conception and design: Z Li; (II) Administrative support: Y Zhao; (III) Provision of study materials or patients: Y Zhao; \\ (IV) Collection and assembly of data: X Hu; (V) Data analysis and interpretation: X Zhao; (VI) Manuscript writing: All authors; (VII) Final approval \\ of manuscript: All authors. \\ "These authors contributed equally to this work. \\ Correspondence to: Xiaogang Zhao. Department of Thoracic Surgery, Shanghai Pulmonary Hospital, Tongji University, No. 507 Zhengmin Road, \\ Shanghai 200433, China. Email: zhangxiaogangsph@163.com.
}

\begin{abstract}
Background: Bronchopulmonary sequestration (BPS) is a rare congenital pulmonary disease. This study aimed to explore the role of uni-portal video-assisted thoracic surgery (VATS) in the surgical treatment of BPS. Methods: Patients who received surgical treatment for BPS in Shanghai Pulmonary Hospital between January 2012 and October 2018 were retrospectively analyzed. Perioperative characteristics, mortality and morbidity were compared between VATS group and control (open surgery) group. Subgroup analysis was further performed in the VATS group.

Results: A total of 89 patients with BPS were reviewed, including 39 males and 50 females with a mean age of 43.9 years (range, 15-71 years). The mean operation time was $142.5 \mathrm{~min}$ (range, 30-345 min), the mean blood loss was $168.9 \mathrm{~mL}$ (range, 20-1,600 mL), the mean duration of chest drainage was $4.6 \pm 1.9$ days in the whole group. There were 21 patients in the uni-portal VATS group, 46 in the multi-portal VATS group and 22 in the control group. Patients in the VATS groups had significantly better outcome, shorter operation time, shorter postoperative hospital stay (POS), less blood loss and less postoperative complications $(\mathrm{P}<0.05)$. The rate of conversion to open surgery in the uni-portal VATS group and multi-portal VATS group was $9.1 \%$ and $8.7 \%$ respectively. Compared with multi-portal VATS group, the operation time, duration of chest drainage and POS were shorter in the uni-portal VATS group, but the incidence of postoperative complications was similar.
\end{abstract}

Conclusions: Uni-portal VATS is a safe, feasible and effective method for selected BPS patients.

Keywords: Uni-portal; video-assisted thoracic surgery (VATS); bronchopulmonary sequestration (BPS); surgical resection

Submitted Oct 29, 2019. Accepted for publication Dec 24, 2019.

doi: $10.21037 /$ jtd.2020.01.32

View this article at: http://dx.doi.org/10.21037/jtd.2020.01.32

\section{Introduction}

Bronchopulmonary sequestration (BPS) is a rare congenital abnormality of the lower airway. It consists of a nonfunctional mass of lung tissues that lack normal communication with the tracheobronchial trees and receive arterial blood supply from the systemic circulation. It accounts for $0.15-6.4 \%$ of respiratory malformations (1). Rare complications of BPS include heart failure due to excessive flow through the aberrant artery, massive bleeding, lung torsion and malignancy caused by chronic stimulation (2). Currently, surgical resection is still the main treatment for the symptomatic BPS.

The conventional surgical approach for BPS is 

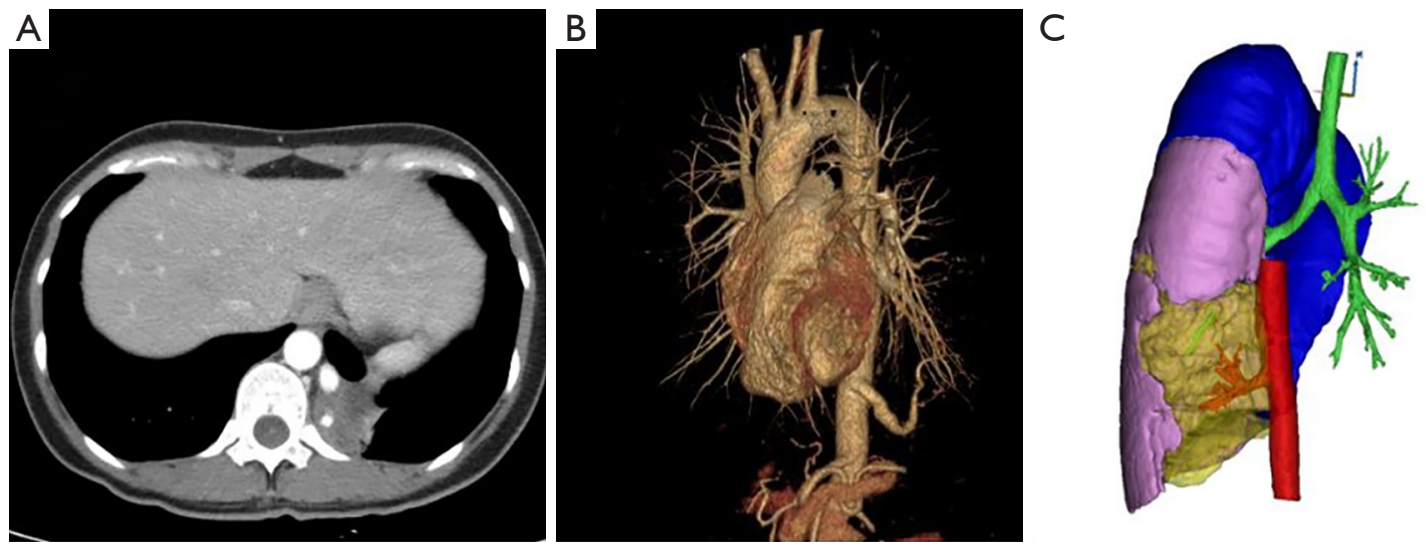

Figure 1 Aberrant artery originating from the thoracic aorta, as shown by CT scanning (A) and 3-dimensional reconstruction (B,C).

posterolateral thoracotomy. Thoracoscopic procedure was once considered to be a contraindication for BPS patients with extensive adhesion caused by chronic infection and complicated unnoticed aberrant artery. However, with the progression of minimally invasive techniques and equipments (3), video-assisted thoracoscopic surgery (VATS) has been widely used in the treatment of BPS, which results in less postoperative pain and faster recovery. In our center, uni-portal VATS was introduced in 2014. In the present study, we retrospectively reviewed a series of patients who received uni-portal VATS for BPS, and the feasibility and safety of uni-portal VATS in the surgical treatment of BPS was assessed.

\section{Methods}

This study was approved by the Institutional Review Board of Shanghai Pulmonary Hospital. Informed consent was obtained from all patients before surgery. BPS patients who underwent surgical treatment in the Department of Thoracic Surgery, Shanghai Pulmonary Hospital between January 2012 and January 2018 were retrospectively analyzed. Demographic characteristics, medical history, CT manifestations, intraoperative findings, and postoperative outcome were collected. The preoperative diagnosis of BPS was based on the medical history and CT manifestations while the diagnosis of BPS was confirmed by the intraoperative findings and postoperative pathology. Detailed preoperative CTA was scanned in every patient and 3-dimensional angiographic imaging was reconstructed in our center if diagnosis of BPS is suspected (Figure 1).

All procedures were performed under single-lung ventilation anesthesia. Thoracotomy was performed by a posterolateral incision. A single incision (about $3 \mathrm{~cm}$ ) was made through the 5 th intercostal space in uni-portal VATS. While in multi-portal VATS, the observation port and operation port were selected in 7 th and 4th intercostal space or 8 th and 5 th intercostal space respectively. All VATS procedures were performed under non-direct view without intercostal rib spreading. Specifically, aberrant arteries were recognized carefully, clamped with clips or hemLock, and ligated with silks or resected by staplers according to the arterial diameter and the preference of surgeons (4). The diameter of each aberrant artery was measured immediately after the sequestrated lesion was retrieved. Pulmonary wedge resection, lobectomy or pneumonectomy was employed for BPS during operation, and the main surgical procedure was lobectomy. A drainage tube was routinely placed after surgery (Figure 2). The drainage tube was removed if following conditions were present: the lungs were well re-expanded, there were no obvious air leaks and bleeding, and the pleural effusion was less than $200 \mathrm{~mL}$ per day. Perioperative outcome included complications or deaths occurring within 30 days after surgery.

Data was analyzed using the SPSS version 16.0 (SPSS Inc., Chicago, IL). Continuous variables with normal distribution were analyzed with $t$-test. Mann-Whitney $\mathrm{U}$ test was performed for variables with abnormal distribution. Categorical variables were compared with Pearson's Chisquare test or Fisher exact test. A value of $\mathrm{P}$ less than 0.05 was considered statistically significant.

\section{Results}

A total of 89 patients who underwent surgical treatment of BPS were included in this study. Right BPS was found 

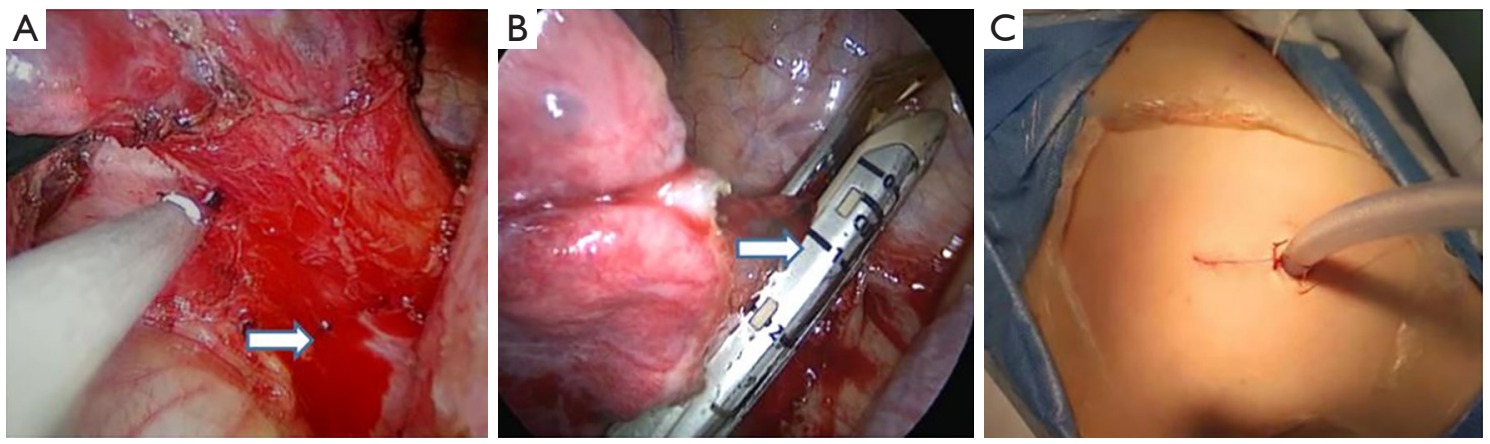

Figure 2 Identification (A) and cutting (B) of the aberrant artery (white arrows) on uni-portal VATS; (C) the single incision and drainage of uni-portal VATS.

in 30 patients, and left BPS in 6. The mean age at surgery was 43.9 years (range, 15-71 years). Most of BPS were ILS (97.7\%). The diagnostic accuracy rate of preoperative CT was $79.8 \%(71 / 89)$, and some patients were misdiagnosed with pulmonary cysts, lung cancer, or other infectious diseases. Preoperative CT revealed a mass in 58.4\% (52/89) of patients, cystic lesions in $37.1 \%(33 / 89)$, and scattered inflammation in $4.6 \%(n=4)$. For the aberrant arteries, most $(82.0 \%)$ originated from the thoracic aorta, and $18 \%$ arose from the abdominal aorta. Most patients $(80.9 \%)$ had 1 single aberrant artery, $11.2 \%$ had 2 aberrant arteries, while $7.9 \%$ had 3 or more aberrant arteries. The diameter of aberrant arteries ranged from 0.2 to $1.4 \mathrm{~cm}$, with a mean diameter of $0.6 \mathrm{~cm}$. In the present study, 21 patients received uni-portal VATS, 46 patients underwent multiportal VATS, and 22 received open-thoracotomy (control group). The rate of major complications in the whole patients was $12.6 \%$ (11/89), and the major complications included postoperative massive bleeding in 1 patient, arrhythmia in 5, pulmonary infection in 4, and air leak in 1 .

The baseline demographic characteristics and perioperative clinical findings in the VATS groups and open surgery group are shown in Table 1 . The patients in the VATS group underwent surgery at a younger age than those in the open surgery group $(41.8 \pm 14.6$ vs. $50.6 \pm 13.3$ years, $\mathrm{P}=0.015)$. The most common preoperative complaints were cough, fever and chest pain. Patients in the open surgery group had more symptoms than those in the VATS group (19/22 vs. 42/67, $\mathrm{P}=0.038)$. Besides the above, there were no significant differences in the gender, preoperative lung function, preoperative CT diagnosis rate, location of BPS, type of BPS, origin of aberrant arteries, and number of aberrant arteries between two groups. Meanwhile, the severity of intraoperative adhesion (no adhesion, local adhesion and extensive adhesion) was also similar between two groups $(\mathrm{P}=0.237)$. In the VATS group, 65 patients underwent anatomic lobectomy. 2 patients received the removal of ELS lesion under sublobar resection. Totally, conversion to thoracotomy during surgery was found in 5 patients (lobectomy), and the causes included extensive adhesion $(n=4)$ and unexpected injury of the aberrant artery resulting in blood loss of $1,600 \mathrm{~mL}(\mathrm{n}=1)$. In the open surgery group, 21 patients underwent anatomic lobectomy. One patient received pneumonectomy due to extensive bronchiectasia in the left upper lobe and widespread cystic BPS in the lower lobe, and 1 underwent excision of the sequestrated lung lesion by sublobar resection. The mean operation time was $126 \mathrm{~min}$ (range, $95-170 \mathrm{~min}$ ) in the VATS group and $132 \mathrm{~min}$ (range, 105-241 min) in the open surgery group $(\mathrm{P}>0.05)$. However, the blood loss in the VATS group was significantly less than in the open surgery group $(\mathrm{P}=0.049)$. In addition, patients in the VATS group had less postoperative complications ( $7 \%$ vs. $27 \%$, $\mathrm{P}=0.014)$, shorter duration of tube drainage $(4.04 \pm 1.46$ vs. $6.41 \pm 2.32 \mathrm{~d}, \mathrm{P}=0.000)$ and shorter hospital stay $(4.61 \pm 1.49$ vs. $7.00 \pm 2.14 \mathrm{~d}, \mathrm{P}=0.000$ ) as compared to those in the open surgery group.

The baseline demographic characteristics and perioperative clinical findings of patients in the uni-portal VATS group and multi-portal VATS group are shown in Table 2. No significances were found in the gender, age, preoperative lung function, preoperative symptoms, preoperative CT diagnosis rate and type of BPS. In addition, there were no marked differences in the location of BPS, type of BPS, CT manifestation of BPS, and source and number of abnormal arteries. The rate of conversion to thoracotomy in the uni-portal VATS group and multiportal VATS group was $9.1 \%$ and $8.7 \%$, respectively. The 
Table 1 Characteristics of patients and BPS before surgery

\begin{tabular}{|c|c|c|c|}
\hline Variable & VATS group & Open surgery group & $\mathrm{P}$ \\
\hline Male & $28(42 \%)$ & $11(50 \%)$ & \\
\hline Female & 39 (58\%) & $11(50 \%)$ & \\
\hline Age (years) & $41.8 \pm 14.6$ & $50.6 \pm 13.3$ & 0.015 \\
\hline FEV1\% & $70.6 \pm 29.1 \%$ & $60.1 \pm 32.3$ & 0.270 \\
\hline Preoperative symptom & & & 0.038 \\
\hline Yes & $42(63 \%)$ & $19(86 \%)$ & \\
\hline None & $25(37 \%)$ & $3(14 \%)$ & \\
\hline Left & 48 (72\%) & $11(50 \%)$ & \\
\hline Right & $19(28 \%)$ & $11(50 \%)$ & \\
\hline Type of lesion & & & 0.283 \\
\hline Intralobar & $65(97 \%)$ & $22(100 \%)$ & \\
\hline Extralobar & $2(3 \%)$ & $0(0 \%)$ & \\
\hline CT manifestation & & & 0.132 \\
\hline Mass & $36(54 \%)$ & $16(73 \%)$ & \\
\hline Cystic lesion & $27(40 \%)$ & $6(27 \%)$ & \\
\hline Number of aberrant arteries & & & 0.229 \\
\hline 1 & $56(84 \%)$ & $16(73 \%)$ & \\
\hline 2 & $8(12 \%)$ & $2(9 \%)$ & \\
\hline$>2$ & $3(4 \%)$ & $4(18 \%)$ & \\
\hline Mean diameter of aberrant artery (cm) & $0.50[0.40-0.90]$ & $0.60[0.30-0.85]$ & 0.866 \\
\hline Extent of adhesion & & & 0.237 \\
\hline No adhesion & $25(37 \%)$ & $4(18 \%)$ & \\
\hline Located adhesion & $31(46 \%)$ & $14(64 \%)$ & \\
\hline Extensive adhesion & $11(17 \%)$ & $4(18 \%)$ & \\
\hline Surgical procedure & & & 0.226 \\
\hline Lobectomy & $65(97 \%)$ & $20(90 \%)$ & \\
\hline Sublobar resection & $2(3 \%)$ & $1(5 \%)$ & \\
\hline Pneumonectomy & $0(0 \%)$ & $1(5 \%)$ & \\
\hline
\end{tabular}

Table 1 (continued) 
Table 1 (continued)

\begin{tabular}{lccc}
\hline Variable & VATS group & Open surgery group & $P$ \\
\hline Operation time (min) & $126[95-170]$ & $132[105-241]$ & 0.113 \\
Blood loss $(\mathrm{mL})$ & $100[50-150]$ & $150[100-325]$ & 0.049 \\
Duration of drainage & $4.04 \pm 1.46$ & $6.41 \pm 2.32$ & 0.000 \\
Postoperative hospital stay & $4.61 \pm 1.49$ & $7.00 \pm 2.14$ & 0.000 \\
Complications & & 6.014 \\
Yes & $5(7 \%)$ & $6(27 \%)$ \\
No & $62(93 \%)$ & $16(73 \%)$ \\
\hline
\end{tabular}

VATS, video-assisted thoracic surgery; FEV1, forced expiratory volume in one second; FEV1\%, forced expiratory volume in one second to forced vital capacity ratio; BPS, bronchopulmonary sequestration.

Table 2 Subgroup analysis of characteristics and BPS in VATS group before surgery

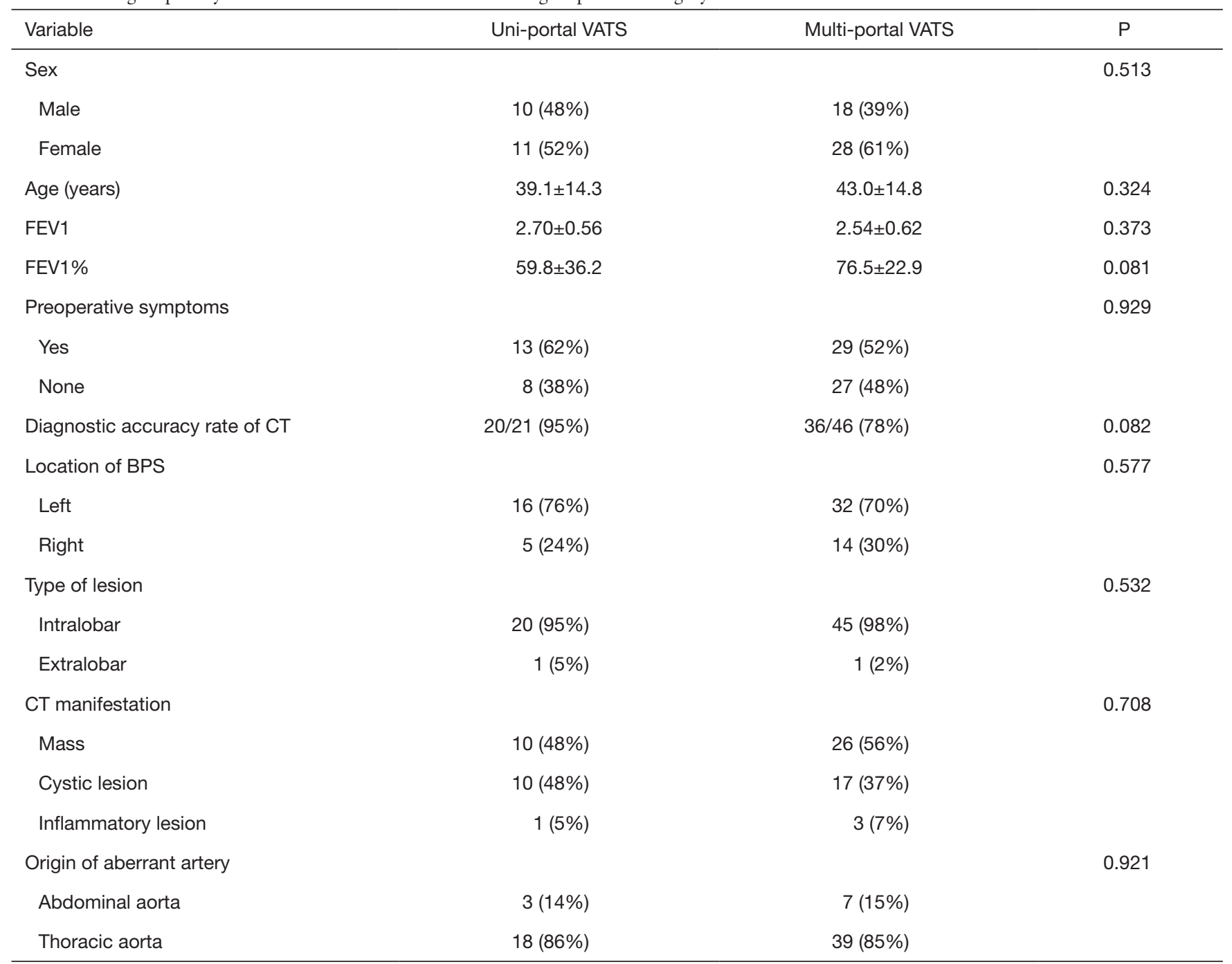

Table 2 (continued) 
Table 2 (continued)

\begin{tabular}{|c|c|c|c|}
\hline Variable & Uni-portal VATS & Multi-portal VATS & $\mathrm{P}$ \\
\hline 1 & $18(86 \%)$ & $38(83 \%)$ & \\
\hline 2 & $3(14 \%)$ & $5(11 \%)$ & \\
\hline$>2$ & $0(0 \%)$ & $3(6 \%)$ & \\
\hline Extent of adhesion & & & 0.074 \\
\hline No adhesion & $12(57 \%)$ & $13(28 \%)$ & \\
\hline Located adhesion & 7 (33\%) & $24(52 \%)$ & \\
\hline Extensive adhesion & $2(10 \%)$ & $9(20 \%)$ & \\
\hline Lobectomy & $21(100 \%)$ & 44 (96\%) & 0.216 \\
\hline Sublobar resection & $0(0 \%)$ & $2(4 \%)$ & \\
\hline Pneumonectomy & $0(0 \%)$ & $0(0 \%)$ & \\
\hline Operation time (min) & $109.0(84.0-130.0)$ & $140.0(112.3-191.2)$ & 0.014 \\
\hline Blood loss (mL) & $100.0(50.0-100.0)$ & $100.0(50.0-200.0)$ & 0.131 \\
\hline Duration of drainage & $3.38 \pm 0.92$ & $4.35 \pm 1.57$ & 0.002 \\
\hline Postoperative hospital stay & $3.81 \pm 0.93$ & $4.98 \pm 1.56$ & 0.000 \\
\hline Complications & & & 0.946 \\
\hline
\end{tabular}

VATS, video-assisted thoracic surgery; FEV1, forced expiratory volume in one second; FEV1\%, forced expiratory volume in one second to forced vital capacity ratio; BPS, bronchopulmonary sequestration.

causes of conversion to thoracotomy included thoracic adhesion and unexpected bleeding. Compared with the multi-portal VATS group, the operation time, duration of postoperative tube drainage and hospital stay in the uniportal VATS group reduced significantly, but there was marked difference in the blood loss between groups. The incidence of complications in the uni-portal VATS group was slightly lower than in the multi-portal VATS group (5\% vs. $9 \% ; \mathrm{P}=0.946)$.

\section{Discussion}

BPS is a congenital lung abnormality, but most BPS patients are asymptomatic at early stage. The clinical symptoms include bronchiectasis, aspergilloma, lung abscess and other pulmonary infections. Since symptoms are often non-specific, the precise diagnosis of BPS is difficult based on medical history, especially in ILS patients with repeated infections of the lower lobe. Currently, due to progression of CT technology, BPS can be diagnosed preoperatively in most patients (5). Preoperative enhanced computed tomographic angiography (CTA) can identify the origin and number of aberrant arteries, providing evidence on the intraoperative management and avoiding unexpected vascular accidents. However, not all the BPS patients receive enhanced CT and/or CTA due to the non-specific symptoms. In a previous case report, the preoperative diagnosis rate of BPS was only $12-58 \%$ (6), which was lower than in our study (79.8\%). Of note, BPS in 18 patients was still not confirmed preoperatively, which 
may result in higher risk for unexpected vascular accidents. According to our experience, preoperative enhanced CT or CTA for acquired lower lobar multi-cystic lesions or atypical bronchiectasis can help improve the identification of BPS.

Surgical resection is still the main treatment for BPS. Thoracoscopic surgery was previously considered inapplicable in the treatment of BPS due to extensive intrathoracic adhesions and difficulty in the identification of aberrant arteries. However, the progresses in the techniques and devices allows the application of thoracoscopic surgery in the treatment of BPS. In the present study, results showed most of the adhesions were local (47.2\%) in BPS patients, and extensive adhesion only accounted for $14.6 \%$. From this aspect, surgical treatment via VATS is feasible and possible for BPS. Some case reports have indicated that VATS has become a mainstream procedure for BPS resection in some experienced centers (7-10). In available study, the rate of conversion to open surgery for BPS ranged from $0 \%$ to $21 \%$, and it in our study was $9.1 \%$ and $8.7 \%$ in the multi-portal VATS group and uni-portal VATS group, respectively.

Lobectomy was most common approach for complete resection of BPS lesion in our study. There is evidence showing that sublobar resection can also achieve satisfactory outcome for localized BPS lesions (11). According to our experience, the surgical approach should be selected mainly depending on the location and size of BPS lesion. Complete removal of BPS lesions and adjacent nonfunctional lung tissues is the key in avoiding recurrence of pulmonary infection. Pneumonectomy is the last choice if there is an uncontrolled and unconfined infection in the ipsilateral cavity because it has high mortality, high morbidity and poor quality of life. In this study, 85 patients underwent lobectomy, 3 received wedge resection, and only 1 underwent pneumonectomy for combined destroyed lung.

For experienced thoracic surgeons, VATS is considered a relatively safe and effective surgical procedure for BPS. The uni-portal VATS is an improvement of the multiportal VATS, and was first reported in 2011 (12). The advantages of uni-portal VATS include less postoperative pain, shorter duration of postoperative drainage, shorter hospital stay, and better appearance as compared to the conventional multi-port VATS $(13,14)$. Uni-portal VATS for BPS is only reported in some case reports (15). To the best of our knowledge, this is the first case report with the largest sample size in which the role of uni-portal VATS in the treatment of BPS was investigated by comparing with the conventional open thoracotomy and multi-portal VATS. There are also obvious technical limitations with uni-portal VATS. It is not a naturally ergonomic procedure, because the traditional thoracoscopic principle of triangulation is omitted. In addition, positioning of multiple devices through a single small incision in the chest poses problems because instruments can often interfere with each other in the pleural and extrapleural spaces. Besides, a singleport up-to-down vision make it difficult to distinguish aberrant arteries, especially when these arteries locate in the pulmonary ligament combined with recurrent infections. Moreover, to achieve adequate angle for stapler to resect the aberrant artery, much more experience and skill are needed in the uni-portal VATS for BPS. However, detailed preoperative CTA or angiographic imaging reconstruction and meticulous management of aberrant arteries during operation may help reduce unexpected intraoperative accidents. In our center (uni-portal VATS accounts for more than $80 \%$ ), uni-portal VATS was introduced for the treatment of BPS lesion resection in 2012. Subgroup analysis of VATS group indicated shorter operation time, similar incidence of perioperative complications, and faster postoperative recovery (shorter duration of drainage and shorter hospital stay) in the uni-portal VATS group as compared to multi-portal VATS group.

However, this study had several limitations: it was a single-center retrospective study with a long span, which may inevitably have selective bias. In addition, patients were not followed up, which makes it impossible to access the improvement of symptoms and the postoperative recurrence. More multi-center prospective clinical trials are needed to further confirm the effectiveness of uni-portal VATS for BPS.

\section{Conclusions}

In conclusion, our retrospective study suggests that lobectomy remains the primary surgical procedure for BPS; uni-portal VATS is safe and feasible for the surgical treatment of BPS, with shorter operation time, shorter hospital stay and lower incidence of perioperative complication. It can be an alternative for multi-portal VATS and thoracotomy for surgical treatment of BPS in an experienced center.

\section{Acknowledgments}

Funding: None. 


\section{Footnote}

Conflicts of Interest: The authors have no conflicts of interest to declare.

Ethical Statement: The authors are accountable for all aspects of the work in ensuring that questions related to the accuracy or integrity of any part of the work are appropriately investigated and resolved. This study was approved by the Institutional Review Board of Shanghai Pulmonary Hospital. Informed consent was obtained from all patients before surgery.

Open Access Statement: This is an Open Access article distributed in accordance with the Creative Commons Attribution-NonCommercial-NoDerivs 4.0 International License (CC BY-NC-ND 4.0), which permits the noncommercial replication and distribution of the article with the strict proviso that no changes or edits are made and the original work is properly cited (including links to both the formal publication through the relevant DOI and the license). See: https://creativecommons.org/licenses/by-nc-nd/4.0/.

\section{References}

1. Kravitz RM. Congenital Malformations of the Lung. Pediatr Clin North Am 1994:41:453-72.

2. Clements BS, Warner JO. Pulmonary Sequestration and Related Congenital Bronchopulmonary-Vascular Malformations: Nomenclature and Classification Based On Anatomical and Embryological Considerations. Thorax 1987;42:401-8.

3. Yue S, Guo H, Zhang YG, et al. The Clinical Value of Computer Tomographic Angiography for the Diagnosis and Therapeutic Planning of Patients with Pulmonary Sequestration. Eur J Cardiothorac Surg 2013;43:946-51.

4. Okamoto J, Kubokura H, Usuda J. Safe Transection of Aberrant Arteries Associated with Pulmonary

Cite this article as: $\mathrm{Li} \mathrm{Z,} \mathrm{Zhao} \mathrm{Y,} \mathrm{Hu} \mathrm{X,} \mathrm{He} \mathrm{W,} \mathrm{Zhao} \mathrm{X.} \mathrm{Is}$ uni-portal video-assisted thoracic surgery a feasible approach for the surgical treatment of bronchopulmonary sequestration? J Thorac Dis 2020;12(3):414-421. doi: 10.21037/jtd.2020.01.32
Sequestrations. BMC Surg 2015;15:27.

5. Kouchi K, Yoshida H, Matsunaga T, et al. Intralobar Bronchopulmonary Sequestration Evaluated by ContrastEnhanced Three-Dimensional MR Angiography. Pediatr Radiol 2000;30:774-5.

6. Wei Y, Li F. Pulmonary Sequestration: A Retrospective Analysis of 2625 Cases in China. Eur J Cardiothorac Surg 2011;40:e39-e42.

7. Kestenholz PB, Schneiter D, Hillinger S, et al. Thoracoscopic Treatment of Pulmonary Sequestration. Eur J Cardiothorac Surg 2006;29:815-8.

8. Reismann M, Gossner J, Glueer S, et al. Thoracoscopic resection of congenital pulmonary malformations in infants: is the feasibility related to the size of the lesion? World J Pediatr 2012;8:272-4.

9. Liu C, Pu Q, Ma L, et al. Video-Assisted Thoracic Surgery for Pulmonary Sequestration Compared with Posterolateral Thoracotomy. J Thorac Cardiovasc Surg 2013;146:557-61.

10. Shen JF, Zhang XX, Li SB, et al. Complete Video-Assisted Thoracoscopic Surgery for Pulmonary Sequestration. J Thorac Dis 2013;5:31-5.

11. Wang LM, Cao JL, Hu J. Video-Assisted Thoracic Surgery for Pulmonary Sequestration: A Safe Alternative Procedure. J Thorac Dis 2016;8:31-6.

12. Gonzalez-Rivas D, Paradela M, Fernandez R, et al. Uniportal Video-Assisted Thoracoscopic Lobectomy: Two Years of Experience. Ann Thorac Surg 2013;95:426-32.

13. Sihoe AD. The Evolution of Minimally Invasive Thoracic Surgery: Implications for the Practice of Uniportal Thoracoscopic Surgery. J Thorac Dis 2014;6:S604-17.

14. Bertolaccini L, Rocco G, Viti A, Terzi A. Geometrical Characteristics of Uniportal VATS. J Thorac Dis 2013;5:S214-6.

15. Tamura M, Shimizu Y, Hashizume Y. Single-Incision Video-Assisted Thoracic Resection for Extrapulmonary Sequestration: A Case Report. J Cardiothorac Surg 2014;9:22. 